\title{
CHARACTERIZATION AND MODELING OF THE FATIGUE BEHAVIOR OF TPU
}

\author{
J. Holzweber ${ }^{1}$, J. Müller ${ }^{2}$, U. D. Çakmak ${ }^{1}$, Z. Major ${ }^{1}$ \\ 1 Johannes Kepler University, Institute of Polymer Product Engineering, Altenberger-Strasse 69, 4040, \\ Linz, Austria. E-mails: juergen.holzweber_1@jku.at,umut.cakmak@jku.at,zoltan.major@jku.at \\ 2 DMH Dichtungs- und Maschinenhandel GmbH, Industriepark West 11, 8772, Traboch, Austria. \\ E-mail: johannes.mueller@dmh.at
}

\section{Introduction}

Thermoplastic polyurethanes (TPU) have a wide range of mechanical and chemical properties due to their various available formulations and getting the material of choice for nowadays applications. The comprehensive determination of the material properties as well as the examination of the long-term fatigue behavior of elastomer composites are of particular practical importance for demanding engineering mechatronic applications.

Contrary to many thermoplastic and metallic materials, rubber and rubberlike materials show a highly nonlinear material behavior in the stressstrain behavior. This characteristic is often linked to hyperelasticity with inherent viscoelasticity. In accordance with Qi and Boyce TPUs are very rate, time- and hysteresis dependent [1]. Especially for materials, exhibiting a strong hyperelastic material response, a throughout characterization of different mechanical loading states are required to gain profound understanding of the mechanical behavior. Based on uniaxial tension, compression, equibiaxial and planar tension tests the complex material behavior can be described and the parameters of more elaborated material models can be reliably determined [2]. Mechatronic applications are usually loaded in non-uniform complex loading states and consequently material parameter determination from a simple tensile test is not adequate. All subsequent analyses, including numerical calculations of components and lifetime estimations, are relying on this.

Therefore, the objectives of this study are:

- The characterization of the complex mechanical material behavior of TPUs under monotonic and cyclic loading.

- The definition of material models and determination of parameters for further FE simulations.
- Application of the local strain based Wöhler curve method for lifetime estimations on a component.

\section{Methods}

One TPU material was selected, characterized and the lifetime of a simplified component was estimated and compared to experimental findings.

\subsection{Monotonic Deformation Behavior}

In the first step the monotonic deformation behavior was characterized. Therefore uniaxial tension and compression, biaxial and planar tests were used. To determine the strong rate and temperature dependence behavior of TPUs, the uniaxial and planar tests were performed with three different rates $(0,1 \mathrm{~mm} / \mathrm{s} ; \quad 1 \mathrm{~mm} / \mathrm{s}$ and $200 \mathrm{~mm} / \mathrm{min}$ ) at three different temperature levels $\left(-30^{\circ} \mathrm{C}, \mathrm{RT}\right.$, and $\left.80^{\circ} \mathrm{C}\right)$.

\subsection{Dynamic Thermomechanical Analysis}

A dynamic characterization with focus on the amplitude-, frequency- and temperature dependency of the modulus was studied by DTMA. These data are used to obtain the Prony Series. According to Tschoegl et al. the principle of the use of this approach is only justified if the material shows a thermorheologically simple behavior. If the loss factor follows the storage modulus as a function, the material is defined as thermorheologically simple and in contrast to the general proposition, the method of the superposition and the fitting of the Prony Series can be used [2],[3].

\subsection{Fatigue Characterization}

To determine the longterm behavior of the TPU in terms of cyclic loadings, displacement controlled fatigue tests at room temperature and a frequency of $5 \mathrm{~Hz}$ at a constant ratio, the quotient of $u_{\min }$ to $u_{\max }$, of 0,1 were performed. The special methodology for the local strain based Wöhler curve starts with displacement controlled tests at 


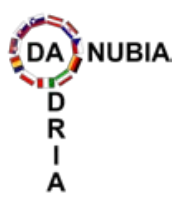

34th Danubia-Adria Symposium on Advances in Experimental Mechanics

University of Trieste, Italy, 2017 different amplitudes. The hysteresis are recorded and the number of cycles to failure are determined. To translate the displacements into local strains a Calibration Curve is necessary. Therefore the local strains of the specimen or the component are evaluated. This can be done experimentally with a DIC system, if possible, or numerically with an FE analysis based on a proper material model obtained from the previous generated material data.

Based on the local strain based Wöhler curve, LSWC, a lifetime estimation on a simplified model of an engine mount was done and compared with experimental results. The material models were calibrated with MCalibration 4.3. The component lifetime was estimated with Abaqus 6.14 and nCode 11 from HBM.

\section{Results}

Fig. 1 presents the experimentally determined Wöhler curve of the TPU including the scattering of the data. The more data are available the better is the reliability of the LSWC curve. The hatched area represents the scattering of the specimens lifetime. The fit of all data points (the green line), generated with the JMP software, is based on a Weibull distribution. This data is used for further lifetime calculations.

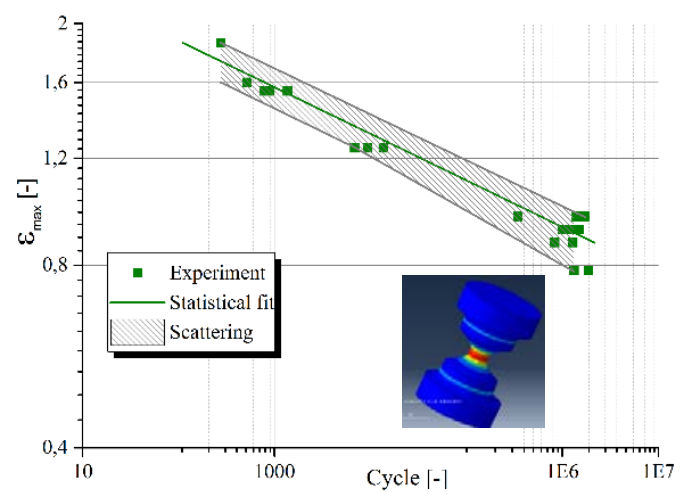

Fig. 1. Experimentally determined Wöhler curve

The results and the deviation between the prediction and the experimental data are shown in Fig. 2. The slopes of both curves in the logarithmic plot are quite the same and the curves are following a similar trend. Nevertheless, there is a deviation between the simulated and the experimental determined results. The deviations seem to get larger in the high cycle region. It should be mentioned that the uniaxial limit as well as the endurance limit was not characterized, neither with the specimen or the component. By the fact that only three components were tested, the scattering of the component tests are not taken into account. However, it should clarify that by fatigue testing and prediction a probability area of failure should always be considered.

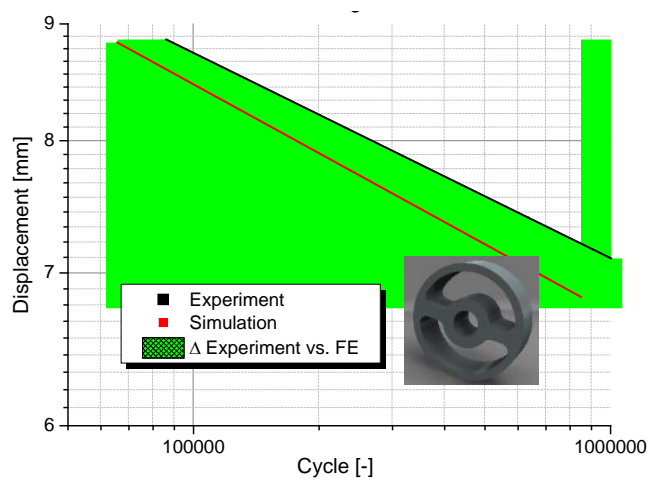

Fig. 2. Deviation between predicted lifetime and experimentally determined lifetime of the component.

\section{Remarks}

- The big variety of the mechanical material testing gives a proper understanding of the complex material behavior of TPUs.

- An adequate material model is essential for further component calculations and for the calibration curve.

- Even if there is a big deviation in the simulated and experimental lifetime, the experimentally determined LSWC can be used as a reference on how the fatigue resistance of the material looks like. A lot of data points are required. Even if fatigue testing is a very time- and costintensive job, for modern engineering applications it becomes more and more indispensable.

\section{References}

[1] Qi, H.J., Boyce, M.C., Stress-Strain behavior of thermoplastic polyurethanes, Mech. Mater, vol.37, 2005. pp. 817-839.

[2] Çakmak, U.D., Major, Z., Experimental Thermomechanical Analysis of Elastomers under Uni- and Biaxial Tensile Stress State, Exp. Mech., vol.54, 2014. pp. 653-663.

[3] Tschoegl, N.W., Knauss, W.G., Emir, I., The Effect of Temperature and Pressure on the Mechanical Properties of Thermo- and/or Piezorheologically Simple Polymeric Materials in Thermodynamic Equilibrium-A critical review, Mech. Time-Depend. Mater., vol.6, 2002. pp. 53-99. 